\title{
Calibração do modelo de aceitação de brechas em interseções urbanas com o microssimulador de tráfego VISSIM
}

\author{
Alessandro Macêdo de Araújo ${ }^{1}$, Manoel Mendonça de Castro Neto ${ }^{2}$ \\ 1 Universidade Federal do Ceará, Departamento de Engenharia de Transportes, alessandro.araujo@det.ufc.br \\ 2Universidade Federal do Ceará, Departamento de Engenharia de Transportes, manoel@det.ufc.br
}

\section{Recebido:}

28 de fevereiro de 2018

Aceito para publicação:

17 de maio de 2018

Publicado:

4 de novembro 2018

Editor de área:

Sara Ferreira

\section{Palavras-chaves:}

Interseções não semaforizadas;

Aceitação de brechas;

Brecha crítica;

Simulação microscópica;

Calibração.

\section{Keywords:}

Two-way STOP-controlled

intersections;

Gap acceptance;

Critical gap; Microscopic simulation;

Calibration.

DOI:10.14295/transportes.v26i3.1603

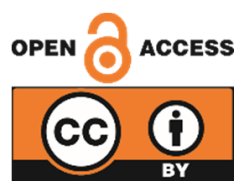

\begin{abstract}
RESUMO
Este artigo tem como objetivo principal propor um método para a modelagem do processo de aceitação de brechas em interseções urbanas não semaforizadas utilizando o microssimulador VISSIM. O método proposto tem como medida-alvo o tempo que o motorista leva no processo de avaliação das brechas, ou seja, o tempo de espera enquanto está na primeira posição da fila. $O \chi^{2}$ da aderência entre as distribuições dos tempos de espera (simulados e observados) e a diferença entre as médias foram utilizados na estimação da brecha crítica. O método foi aplicado em uma interseção da cidade de Fortaleza. Os resultados indicaram valores levemente diferentes da brecha crítica entre os movimentos de conversão. O segundo objetivo deste trabalho é comparar o método proposto com o método de calibração mais convencional, no qual a medida-alvo é uma medida de desempenho do tráfego, como o tamanho médio da fila. $O$ método proposto apresentou melhores resultados.
\end{abstract}

\begin{abstract}
The main objective of this paper is to propose a method to model gap acceptance in urban two-way STOP-controlled intersections using VISSIM microscopic simulator. The proposed method is based on the time that the driver takes in the process of gap acceptance, that is, on the time that the vehicle stays in the first position of the queue. The $\chi^{2}$ of the adherence between the waiting times' distributions (simulated and observed) and the difference between the means were used for the critical gap estimation. The proposed method was applied to an intersection located in Fortaleza city. The results indicated slightly different critical gap values between turning movements. The second objective of this paper is to compare the proposed calibration procedure with the conventional one, in which the calibration target is a traffic measure of effectiveness, such as the average queue length. The proposed method yielded better results.
\end{abstract}

\section{INTRODUÇÃO}

A modelagem do tráfego urbano tem como objetivo primordial representar a formação e a descarga das filas nas interseções. Para interseções não semaforizadas (INS), as filas nas aproximações secundárias dependem fortemente do comportamento de aceitação das brechas da via principal pelos motoristas da via secundária. Esse processo de decisão varia, dentre outros fatores, com a percepção de cada condutor (Garber e Hoel, 2015) e com o tipo de conversão (Brilon et al., 1999; TRB, 2010; Elefteriadou, 2014), e é a partir dele, juntamente com a demanda veicular na interseção e suas características físicas, que o comportamento da fila é modelado.

O software de microssimulação VISSIM versão 9.0 (PTV, 2016) disponibiliza dois modelos de 
aceitação de brechas: o conflict areas, ou áreas de conflito (AC), cuja abordagem é mais complexa, com maior quantidade de parâmetros ajustáveis; e o priority rules, ou regras de prioridade (RP). Independente do modelo selecionado, a sua calibração é condição essencial para a estimação adequada das medidas de desempenho do tráfego.

Comumente, a calibração de parâmetros de um modelo de microssimulação tem como medida-alvo (medida cujos erros entre os valores simulados e os observados em campo são minimizados) a própria medida de desempenho do tráfego que se pretende estimar, como o tempo de viagem, o atraso veicular, o comprimento de fila ou a capacidade (Liu et al., 2012; MartinGasulla et al., 2017; Lidbe et al., 2016). A grande desvantagem desse procedimento de calibração é que essas medidas de desempenho podem ser bastante sensíveis a outras variáveis que não estão diretamente relacionadas ao processo de aceitação de brechas, como a própria demanda na via secundária e o espaçamento entre os veículos quando estão em fila. Portanto, se esses e outros fatores não estão bem representados no simulador, os valores calibrados para os parâmetros do modelo de aceitação de brechas provavelmente não serão adequados, pois a calibração tentará compensar os erros de representação desses fatores por meio de valores irreais dos parâmetros.

Com base no que foi exposto, o objetivo principal deste trabalho é propor um método para modelagem do processo de aceitação de brechas em interseções não semaforizadas urbanas, com uso do microssimulador VISSIM. Um segundo objetivo deste trabalho é comparar a calibração proposta com a calibração que tenha como medida-alvo uma medida de desempenho do tráfego, como tamanho médio de fila, abordagem essa mais comum.

\section{MODELAGEM DE ACEITAÇÃO DE BRECHAS}

A etapa inicial da representação do tráfego em uma INS consiste na modelagem da aceitação de brechas, cujos parâmetros principais são a brecha crítica e o tempo de follow-up. Basicamente, existem três formas de se estimar os valores desses parâmetros. A primeira se dá por meio da estimativa em campo de uma variável fortemente relacionada com o conceito de brecha crítica. Um exemplo é o método proposto por Raff (1950), que define a brecha crítica como sendo o intervalo de tempo tal que a quantidade de brechas aceitas com duração menor que ele é igual à de brechas rejeitadas com duração maior que ele.

A segunda forma de estimação se dá por modelos empíricos como os propostos pelo HCM2010 (TRB, 2010), nos quais os valores de headway crítico (no caso do HCM2010 se utiliza esse conceito em vez do de brecha crítica por simplificar o esforço de coleta, já que requer apenas os instantes de passagem da dianteira de cada veículo) e tempo de follow-up são função das características da interseção e da demanda, como geometria, tipo de conversão analisado, greide e porcentagem de veículos pesados. Uma vantagem de modelos desse tipo é que os mesmos vêm sendo calibrados e validados há bastante tempo, embora primordialmente no contexto norte-americano. Porém, além da inexistência de esforços de validação e de adaptação para a realidade brasileira, esses modelos se limitam a situações padrões que podem não representar satisfatoriamente as particularidades do cruzamento em estudo (Tarko e Tian, 2003; Depiante e Galarraga, 2012), como a suposição de que os headways na via principal seguem a distribuição de probabilidade exponencial negativa.

A terceira maneira se dá por microssimulação, que se fundamenta principalmente nas características comportamentais dos indivíduos e permite modelar diversas configurações geométricas e operacionais. Nessa abordagem, a brecha crítica é estimada por tentativa e erro, tendo 
como objetivo a minimização dos erros de estimação de uma medida-alvo. São apresentados a seguir alguns detalhes sobre a modelagem de aceitação de brechas no simulador microscópico VISSIM.

\subsection{Modelagem de aceitação de brechas no VISSIM}

Como dito anteriormente, o VISSIM oferece dois modelos de aceitação de brechas: o regras de prioridade (RP), que considera basicamente um parâmetro relacionado à brecha crítica; e o áreas de conflito (AC), que utiliza nove parâmetros para simular a antecipação dos condutores no processo de aceitação das brechas. No modelo AC, é incorporado também o nível de colaboração dos motoristas da via principal na não obstrução da interseção e na desaceleração frente aos conflitos, corroborando as conclusões obtidas por Mohan e Chandra (2016) a respeito da influência dos veículos da via secundária sobre o fluxo principal em cruzamentos não semaforizados.

Apesar de ser possível evitar a obstrução da interseção utilizando o RP, somente o modelo AC permite a especificação da proporção dos motoristas da via principal que buscam não bloqueá-la quando há congestionamento. Também apenas o modelo AC incorpora a questão da visibilidade na interseção, mas de tal forma que a partir de determinada posição na aproximação os condutores possuem plena visão da corrente de tráfego conflitante. A aceitação de brechas no modelo áreas de conflito é governada principalmente por dois parâmetros que representam folgas entre o veículo da aproximação secundária e os veículos da via principal que devem ser satisfeitas simultaneamente; porém, apesar de relacionadas com o conceito, essas margens de segurança não representam a brecha crítica, que é o parâmetro mais usado na literatura sobre modelagem do processo de aceitação de brechas.

Apesar de ser mais simples, o modelo RP possui os principais elementos necessários para a modelagem do tráfego em cruzamentos urbanos não semaforizados típicos, onde a distância de visibilidade é pequena, ou seja, os motoristas da via secundária precisam se aproximar bastante da interseção para conseguirem observar o fluxo conflitante, anulando assim os benefícios em se utilizar o AC para este quesito. Além disso, o principal parâmetro do modelo regras de prioridade é muito semelhante ao conceito de brecha crítica. Essas talvez sejam as razões pelas quais na literatura são encontrados apenas trabalhos de calibração utilizando o modelo RP, com destaque para Liu et al. (2012) e Viti et al. (2013). Alguns dos atributos do modelo RP estão descritos a seguir e mostrados na Figura 1:

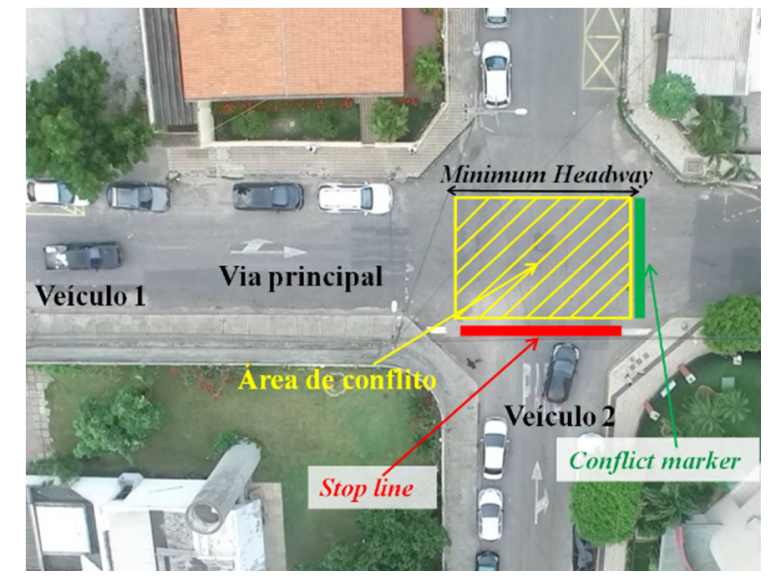

Figura 1: Interseção não semaforizada modelada com o modelo RP do VISSIM 
- Conflict marker: é a seção de referência para determinação da brecha disponível;

- Stop line: é a seção que define o local de parada dos veículos nas aproximações secundárias;

- Min_gap_time: é o mínimo intervalo de tempo, entre o conflict marker e o veículo mais próximo que está trafegando em direção a ele, para que a brecha seja aceita;

- Minimum headway (de espaço): é a mínima distância entre o conflict marker e o veículo da via principal (veículo 1) a montante para que seja permitida a passagem do veículo da aproximação secundária (veículo 2). Ou seja, esse parâmetro impede a entrada do veículo 2 na área de conflito quando ela estiver ocupada e, dessa forma, impacta basicamente em condições de congestionamento da via principal; e

- Maximum speed: é o limite de velocidade acima da qual os veículos da via principal não são considerados na avaliação do minimum headway de espaço. Ou seja, mesmo se o headway de espaço disponível não for aceitável, o veículo 2 irá atravessar a interseção caso o veículo 1 esteja trafegando a uma velocidade maior que a definida pelo parâmetro e se a brecha disponível for maior que o min_gap_time.

\subsection{Calibração de modelo de aceitação de brechas do VISSIM}

$\mathrm{Na}$ literatura existem alguns trabalhos nos quais foram apresentados métodos de calibração de modelo de aceitação de brechas do VISSIM, como o de Liu et al. (2012), onde o min_gap_time foi calibrado simultaneamente com os parâmetros velocidade média na conversão e proporção de escolha por cada faixa de destino de veículos que realizam retorno em interseções não semaforizadas, tendo como medida-alvo a capacidade do movimento, com dados de 13 interseções. 0 modelo calibrado resultou em um erro percentual absoluto médio de 17,6\%, para vias com 4 faixas (min_gap_time de 6,3 segundos obtido), e 20,7\%, para vias com 6 faixas (min_gap_time de $5,1 \mathrm{~s}$ ), na própria capacidade do movimento. Portanto, mesmo a medida-alvo sendo a medida de desempenho que se buscava estimar, o erro obtido foi considerável.

Martin-Gasulla et al. (2017) também utilizaram o modelo RP e calibraram simultaneamente a posição das stop lines e dos conflict markers, a extensão de área de redução de velocidade, a condição dinâmica da fila formada, o number of observed vehicles e o look ahead distance (esses últimos dois pertencem ao modelo de car-following) com a finalidade de microssimular o tráfego de uma faixa em rotatórias localizadas na Espanha. A medida-alvo adotada foi o tamanho médio da fila nas aproximações das rotatórias, com critério de ajuste igual a 15\%. A brecha crítica foi estimada através das filmagens em campo utilizando-se diversos métodos e amostragens diferentes, obtendo-se valores entre 2,6 e 4,7 segundos, que foram inseridos no modelo (min_gap_time) a fim de estimar a capacidade de uma aproximação da rotatória. A estimativa da capacidade obtida pelo modelo calibrado foi melhor que a obtida por diversos modelos analíticos avaliados, porém nenhuma medida de erro para essa variável foi apresentada (apenas ilustrações gráficas).

Calibrações simultâneas podem resultar em estimativas irreais dos parâmetros, já que diferentes combinações de valores podem gerar erros de calibração baixos. Sugere-se neste trabalho utilizar medidas-alvo que isolem o efeito de fatores externos. Para o caso em questão, devese selecionar uma medida que dependa fortemente dos valores da brecha crítica e que não dependa, ou dependa pouco, de outros fatores.

Uma busca por esse tipo de calibração foi apresentada por Viti et al. (2013), que utilizaram 
como medida-alvo para calibrar o min_gap_time o tempo de viagem necessário para os veículos da aproximação secundária se inserirem no fluxo principal, cronometrado a partir do local onde o veículo se posiciona para esperar por uma brecha aceitável até uma seção a jusante localizada longe o suficiente para os veículos completarem sua fase de aceleração. Porém, vale salientar que, apesar dessa medida-alvo não depender fortemente da demanda da via secundária, ela possui a desvantagem de depender dos perfis de aceleração dos veículos. Viti et al. (2013) também ajustaram algumas funções que relacionam o min_gap_time com a demanda da via principal e obtiveram valores de 2,6 a 5,3 segundos para diferentes condições de tráfego em 5 interseções não semaforizadas em forma de "T" localizadas na Lovaina, Bélgica, totalizando 11 casos de estudo. Compararam-se os resultados obtidos pela calibração de diferentes modelos (funções mencionadas acima) e os melhores resultados foram obtidos com o modelo exponencial proposto pelos autores.

\section{MÉTODO EMPREGADO}

0 método adotado neste trabalho é composto de três fases. A primeira consistiu na compreensão do modelo RP, com base em revisão bibliográfica e em simulações preliminares que incluíram análises de sensibilidade dos parâmetros.

A segunda fase do método teve como objetivo principal estabelecer a estratégia de calibração $e$ de validação do modelo. Inicialmente foram escolhidos os parâmetros a serem calibrados, como também a medida-alvo de calibração que isolasse os efeitos de outros fatores e cuja coleta fosse viável. Em seguida, foram estabelecidos os critérios para avaliar a qualidade do ajuste para a calibração e validação, podendo ser uma simples medida de erro ou um teste estatístico para verificar a aderência dos dados observados em campo à distribuição de probabilidade que geraram os dados simulados.

A terceira e última fase do método foi a realização da calibração e da validação do modelo. 0 primeiro passo desta fase foi a coleta dos dados de demanda, da medida-alvo e da infraestrutura. Antes da calibração, recomenda-se realizar a comparação entre as distribuições de frequência modelada no simulador e observada em campo dos headways (ou das brechas) da aproximação principal, já que a aceitação de brechas é bastante dependente do padrão de chegada dos veículos da via principal (Kyte et al., 2003; TRB, 2010; Elefteriadou, 2014). Para a comparação entre os valores simulados e os observados em campo na fase de validação, propõe-se o uso de intervalos de confiança (IC) em vez de testes de hipóteses de igualdade das médias, pois o IC além de oferecer o resultado do referido teste de hipótese, ele também oferece uma estimativa intervalar para a diferença; ou seja, além da significância estatística, ele apresenta a significância prática. 0 procedimento de calibração e validação foi aplicado separadamente para cada movimento proveniente da aproximação secundária, já que o tipo de conversão é um dos fatores que influenciam o processo de aceitação de brechas (TRB, 2010; Elefteriadou, 2014).

Ao final, o método de calibração proposto foi comparado com o método convencional, em que a medida-alvo é a medida de desempenho do tráfego que se deseja estimar, como tamanho médio de fila ou atraso. Para o desenvolvimento deste trabalho, foi criada uma rotina computacional em linguagem de programação Python, que possui fácil acesso e manipulação aos objetos e funções do VISSIM por meio de interface COM.

\section{APLICAÇÃO DO MÉTODO E RESULTADOS}

Para melhor estimar a brecha crítica na aproximação secundária, optou-se por aplicar o método 
proposto em uma interseção cujo fluxo principal chegasse à interseção de forma mais aleatória possível, pois a chegada em pelotões permitiria que os veículos da via secundária se inserissem apenas após a passagem de todo o grupo e, portanto, pouco dependeria da brecha crítica dos condutores. Na escolha da INS, tomou-se como referência o banco de dados contendo a localização dos semáforos de Fortaleza. A INS selecionada foi a do cruzamento entre as ruas Pereira Valente (via principal) e Coronel Linhares, ambas de sentido único, indicada na Figura 2 pelo sinal de "Pare"; nela também são mostrados os semáforos mais próximos dessa interseção.

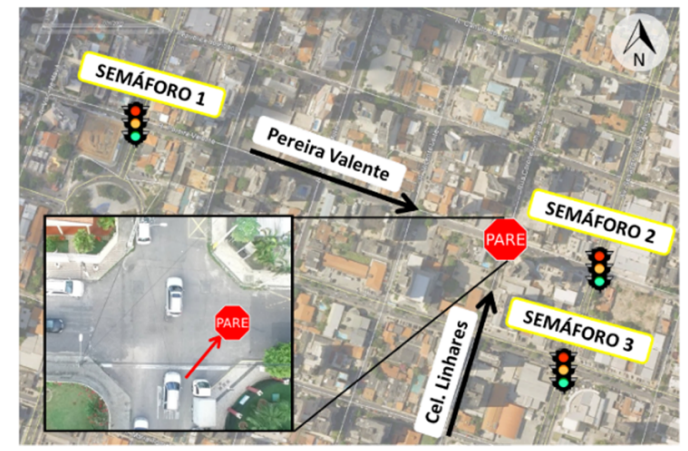

Figura 2: Interseção não semaforizada do estudo de caso

\subsection{Compreensão do modelo RP}

A INS escolhida e os semáforos mais próximos que influenciam na operação dela foram codificados, exceto o semáforo 1, pois foi observado que a chegada dos veículos na Rua Pereira Valente não ocorre em pelotões. Isso acontece porque ela é uma via secundária em alguns dos cruzamentos a jusante do semáforo 1 e devido à entrada de veículos provenientes dessas outras interseções, tornando a chegada dos veículos da aproximação principal com padrão mais aleatório. As programações semafóricas foram definidas conforme os dados cedidos pelo Controle de Tráfego em Área de Fortaleza (CTAFOR).

Com base na definição dos parâmetros apresentada no manual da PTV (2016), o parâmetro min_gap_time é o principal do modelo RP a influenciar a operação de interseções não semaforizadas onde não há congestionamento na via principal, como é o caso da interseção em estudo. Tal fato foi confirmado com uma análise de sensibilidade do tipo um-por-vez dos outros dois parâmetros, minimum headway (variando seu valor entre 2,0 m e 8,0 m em torno do valor default de 5,0 m) e maximum speed (variando entre $20 \mathrm{~km} / \mathrm{h}$ e $180 \mathrm{~km} / \mathrm{h}$, sendo este o seu valor default), na interseção do estudo de caso, da qual se pôde concluir que não houve efeito algum do minimum headway na estimativa do atraso, do comprimento de fila nem da capacidade da aproximação secundária (com os demais parâmetros em seus valores default), enquanto o impacto do maximum speed foi perceptível apenas para valores inferiores às velocidades desejadas dos veículos e o do min_gap_time foi, como esperado, bastante evidente. Apesar da influência do maximum speed sob essas condições, seu valor default é bastante elevado justamente porque sua aplicação simula a entrada forçada (ou o cuidado) dos condutores em casos de congestionamento na área de conflito.

\subsection{Desenvolvimento do método de calibração e validação}

A partir do que foi compreendido com base na revisão bibliográfica e no entendimento da aplicabilidade do modelo RP para a interseção em estudo, iniciou-se o desenvolvimento do método de calibração do parâmetro min_gap_time. 
A medida-alvo proposta neste trabalho para se calibrar o min_gap_time é composta basicamente pelo tempo de espera do veículo enquanto avalia as brechas da via principal, ou seja, o tempo de espera enquanto o veículo está na primeira posição da fila - doravante denominada apenas como "tempo de espera" -, mas também inclui o tempo que o veículo leva para percorrer a sua própria extensão, aumentando a precisão e objetividade da medição. Esta medida-alvo difere da utilizada por Viti et al. (2013) porque o instante inicial do intervalo de tempo é definido pela passagem da frente do veículo por uma seção de referência localizada na aproximação secundária e o instante final é determinado pela passagem da traseira dele pela mesma seção, como ilustrado na Figura 3. A utilização do tempo de espera como medida-alvo diminui significativamente a influência da aceleração dos veículos na estimativa do min_gap_time, fazendo com que a medida-alvo escolhida seja afetada basicamente apenas pelo parâmetro a ser calibrado, o que é desejável.

A seção da faixa de retenção foi tomada como referência para as medições do tempo de espera em campo, pois a sua marcação no pavimento facilita a identificação dos instantes que definem o intervalo a ser registrado. No caso dos poucos veículos que pararam antes dela, a seção de referência considerada foi aproximadamente aquela na qual se atingiu a velocidade nula.

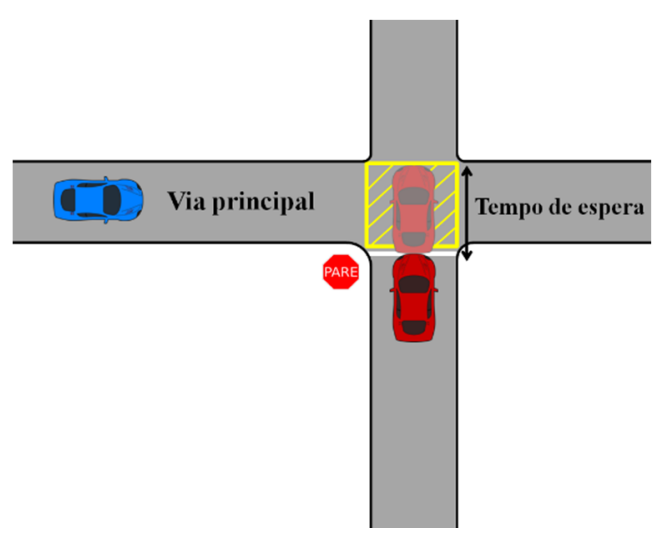

Figura 3: Medida-alvo adotada neste trabalho

Na simulação, a localização da seção de referência para obtenção dos dados do tempo de espera (data collection points) foi escolhida de forma que os veículos da via secundária, ao avaliarem as brechas, parem sobre ela. Tal seção não poderia coincidir com a faixa de retenção (stop line) do modelo porque a maioria dos veículos para a montante dela. Em contrapartida, a seção não poderia ser posta muito antes porque o tempo de espera começaria a ser contabilizado precocemente.

Os tempos de espera obtidos no simulador foram ajustados a fim de descontar parte do tempo de desaceleração dos veículos da via secundária contabilizado nos dados modelados, pois foi observado que no modelo esse processo de desaceleração ocorre de maneira mais demorada. 0 valor desse ajuste foi estimado de tal forma que, ao subtraí-lo das observações simuladas, a diferença absoluta entre as médias da medida-alvo obtida em campo e no modelo fosse mínima, considerando apenas os veículos que pararam momentaneamente e aceitaram a primeira brecha disponível, representando assim uma situação que depende exclusivamente de fatores externos ao processo de aceitação de brechas. 0 intuito dessa correção é tornar as observações simuladas equiparáveis às obtidas em campo, evitando a superestimação causada 
pelo tempo excessivo de desaceleração dos veículos computado em forma de tempo de espera modelado, além do efeito de outros fatores como o tempo de reação dos motoristas e a própria localização da seção de referência.

A amostra de campo dos tempos de espera referentes à situação de parada momentânea, desconsiderando os que foram agressivos a ponto de apenas desacelerar mas sem parar, foi estabelecida com base nas filmagens, enquanto a amostra dos tempos mínimos de espera modelados foi obtida a partir dos resultados das simulações do modelo da interseção modificado apenas quanto à ausência de demanda na aproximação principal. 0 ajuste necessário foi então estimado pela diferença entre as médias dessas duas amostras, para cada movimento de conversão, que foi de 2,3 s para o movimento seguir em frente e 2,1 s para a conversão à direita.

Tanto para a verificação dos headways na via principal quanto na calibração e na validação do modelo, as distribuições observada em campo e simulada da variável em questão (headway ou tempo de espera) foram comparadas usando a estatística $\chi^{2}$, com nível de significância de $5 \%$.

Cabe ressaltar um importante contraponto ao que é comumente encontrado na literatura, onde as frequências da distribuição dos dados observados em campo são consideradas como as frequências esperadas (Jie et al., 2013) no cômputo da estatística $\chi^{2}$. Neste artigo sugere-se utilizar as frequências segundo as classes da distribuição dos dados simulados como as frequências esperadas, pois o teste de aderência deve avaliar se os dados observados em campo podem ser considerados uma amostra da distribuição de probabilidade da variável gerada pelo modelo de simulação. 0 mesmo acontece quando se testa se uma variável pode ser bem representada por uma distribuição de probabilidade: tomam-se as frequências segundo o modelo de probabilidade como as frequências esperadas, e não o contrário. Além disso, tratar as frequências simuladas como esperadas possibilita a obtenção de quantas observações forem desejadas, obtendo-se assim frequências esperadas mais representativas da população definida pelo modelo.

Para a calibração e validação, estimou-se a diferença entre as médias dos valores simulados e observados da variável tempo de espera, sendo a validação apoiada pela estimação intervalar dessa diferença, com grau de confiança de 95\%.

\subsection{Coleta de dados e codificação da interseção de estudo}

Conforme o método de calibração e validação estabelecido, foram coletadas as variáveis headways entre os veículos da via principal, os tempos de espera e os volumes nas duas aproximações. 0 tráfego na interseção foi filmado por meio de um drone do modelo Phantom 3, em dois dias, totalizando uma hora de vídeo, e por meio de câmeras, em outros dois dias, com duração de aproximadamente $1 \mathrm{~h}$ em cada, sendo uma parte descartada (condições diferentes) e metade do restante destinada à calibração e a outra metade à validação do modelo. As filmagens ocorreram nos dias 18 de janeiro e 01 de março de 2016, 25 de maio de 2017 e 27 de março de 2018 (segunda, terça, quinta e terça-feira, respectivamente), entre $16 \mathrm{~h}$ e $17 \mathrm{~h}$.

Para inserção dos dados de demanda no modelo, foram coletados os fluxos direcionais e classificatórios nas duas aproximações, exibidos na Tabela 1. Nota-se que ambas as amostras de dados (calibração e validação) possuem características semelhantes, com a demanda da via principal aproximadamente $10 \%$ superior e o fluxo da via secundária $10 \%$ inferior no cenário de validação, além de pequenas diferenças nas proporções de conversão e no percentual de veículos de médio ou grande porte. 
Tabela 1: Demanda de tráfego nas aproximações da INS

\begin{tabular}{|c|c|c|c|c|}
\hline \multirow{2}{*}{ Etapa } & \multirow{2}{*}{ Variável } & \multicolumn{2}{|c|}{ Aproximação principal } & \multirow{2}{*}{$\begin{array}{c}\text { Aproximação secundária } \\
\text { Total, exceto motos }\end{array}$} \\
\hline & & Total, exceto motos & Motos & \\
\hline \multirow{3}{*}{ 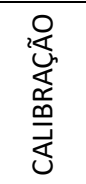 } & Fluxo (veíc/h) & 490 & 67 & 399 \\
\hline & Proporção de conversão & $20 \%$ & $15 \%$ & $36 \%$ \\
\hline & $\begin{array}{c}\text { Proporção de caminhões pequenos } \\
\text { ou vans }\end{array}$ & $0,5 \%$ & - & $2,7 \%$ \\
\hline \multirow{3}{*}{ 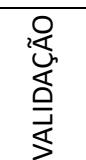 } & Fluxo (veíc/h) & 545 & 61 & 359 \\
\hline & Proporção de conversão & $25 \%$ & $19 \%$ & $32 \%$ \\
\hline & $\begin{array}{l}\text { Proporção de caminhões pequenos } \\
\text { ou vans }\end{array}$ & $1,6 \%$ & - & $1,5 \%$ \\
\hline
\end{tabular}

Os valores considerados para o dado de entrada desired speed foram obtidos dos estudos de Lacerda e Castro-Neto (2014) e Lacerda (2016), que foram coletados em vias de Fortaleza localizadas relativamente próximas ao cruzamento de estudo, enquanto os demais parâmetros comportamentais do VISSIM, exceto o min_gap_time, foram mantidos em seus valores default.

\subsection{Calibração e validação do modelo de aceitação de brechas}

Como mencionado anteriormente, a verificação do padrão de chegada dos veículos do fluxo principal é fundamental devido à sua grande influência no processo de aceitação de brechas. A comparação entre as distribuições simulada e observada dos headways foi feita pelo teste $\chi^{2}$ com nível de significância de $5 \%$ e nove classes. $0 \chi^{2}$ obtido $(4,3)$, com o min_gap_time ainda em default (não exerce influência no padrão de chegada), foi inferior ao valor crítico de 15,5, portanto a hipótese de que a amostra coletada em campo possui a mesma distribuição de probabilidade gerada pelo modelo não foi rejeitada, o que permitiu prosseguir com a calibração do modelo de aceitação de brechas.

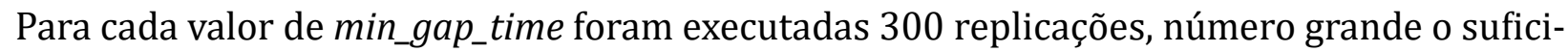
ente para incorporar a variabilidade do modelo e ao mesmo tempo estabilizar a distribuição das variáveis envolvidas (comparada com a amostra de campo, tem-se uma amostra 300 vezes maior de tempos de espera e de headways), com duração de 30 minutos cada, mais dois minutos de warm-up. Esse número de replicações gerou erros de estimação da média dos tempos simulados de no máximo 0,1 s, para um grau de confiança de $95 \%$.

As curvas obtidas para a estatística $\chi^{2}$ e para a diferença entre as médias amostrais da medida-alvo de campo e simulada correspondentes a cada movimento de conversão da rua Cel. Linhares são mostradas na Figura 4. No cálculo dessas médias, foram desconsiderados os veículos que pararam apenas momentaneamente, tanto porque as durações desses tempos de espera são menos influenciadas pelo valor do parâmetro e representam, no caso dessa INS, uma grande parcela das observações, quanto porque as médias dessa classe já foram compatibilizadas devido ao ajuste nos valores simulados.

Dentre todos os valores de min_gap_time testados para o movimento de seguir em frente, os menores que 3,5 s corresponderam a um $\chi^{2}$ superior ao crítico, que neste caso é igual a 11,1 ( $\alpha$ $=5 \%$ e $\mathrm{gl}=6$ ), o que ocorreu principalmente porque os $\chi^{2}$ parciais das últimas classes foram superestimados devido à baixa frequência modelada nesses casos. Além disso, esses cenários corresponderam a uma diferença absoluta elevada entre as médias dos tempos de espera. Para

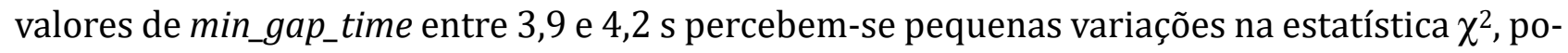


rém não insignificantes para a diferença entre as médias. Com base em ambos os critérios, adotou-se o valor de 4,1 s para o parâmetro (em destaque na figura), correspondendo ao menor $\chi^{2}$ $(0,6)$ e a uma pequena diferença absoluta entre as médias amostrais simulada e observada $(0,2$ segundos).

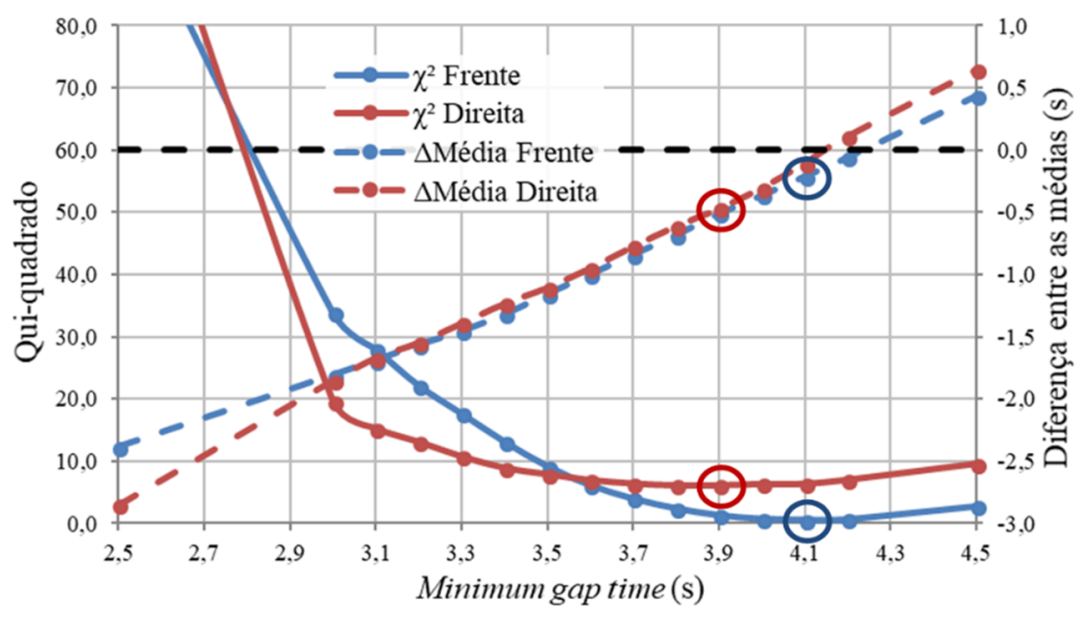

Figura 4: Calibração pelo método proposto

De forma análoga, realizou-se a calibração do parâmetro para a conversão à direita. Dentre os cenários avaliados para esse movimento, os valores abaixo de 3,3 s e o valor de 4,5 s corresponderam a um $\chi^{2}$ superior ao crítico (igual a 9,5), além de terem apresentado as maiores diferenças absolutas entre as médias da medida-alvo. Os menores valores de $\chi^{2}$ foram obtidos com min_gap_time entre 3,6 e 4,2 s, sendo o valor de 3,9 s o que resultou no menor $\chi^{2}(6,1)$ e a uma pequena diferença absoluta entre as médias amostrais $(0,5 \mathrm{~s})$. Atribuindo-se maior peso à comparação entre as distribuições e considerando que 0,5 s de diferença absoluta entre as médias não é muito relevante, adotou-se 3,9 s como o valor calibrado para o parâmetro. 0 valor ótimo nesse caso foi levemente inferior ao obtido para o movimento de seguir em frente, em acordo com o esperado (TRB, 2010). Isso possivelmente acontece porque a área de conflito no caso da conversão é menor, ocorrendo principalmente na faixa de tráfego mais próxima.

Para a validação do modelo foram obtidas as três distribuições dos tempos de espera do primeiro veículo da fila mostradas na Figura $5(\mathrm{~N}=229$ observações): coletada em campo, obtida pelo modelo com o min_gap_time calibrado $(4,1 \mathrm{~s})$ e a com o parâmetro com valor default $(3,0$ s), para o movimento seguir em frente.

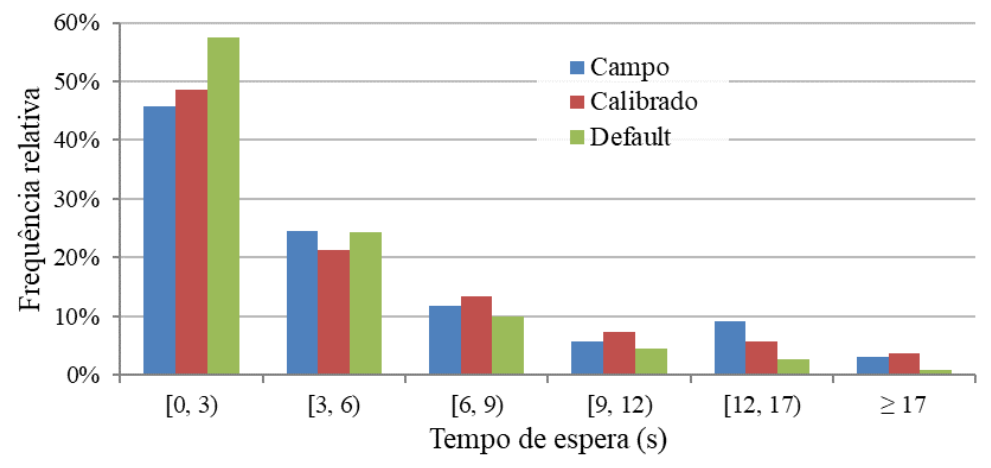

Figura 5: Validação - movimento seguir em frente 
Como é possível perceber, a aderência entre a distribuição de campo e a com o parâmetro calibrado foi satisfatória, correspondendo a uma estatística $\chi^{2}$ de validação igual a 7,9 e resultando na não rejeição da hipótese nula de que a amostra observada pode ser bem representada pelo modelo de simulação; já a distribuição com valor default se distanciou mais, principalmente nas classes das extremidades, cuja hipótese nula foi rejeitada com $\chi^{2}$ de 55,1. A média dos tempos de espera simulados pelo modelo calibrado foi de 8,4 s, enquanto a média obtida em campo foi de $8,1 \mathrm{~s}$, com um intervalo de confiança de $95 \%$ para a diferença entre as médias igual a [-1,2 s; 0,5 s]. Portanto, há indícios que as médias dos tempos de espera modelados e observados são muito similares.

As distribuições da medida-alvo obtidas para a conversão à direita são apresentadas na Figura 6 ( $\mathrm{N}=107$ observações). Nesse caso, a aderência entre as distribuições observada em campo e simulada com parâmetro calibrado foi melhor do que para o movimento de seguir em frente, com $\chi^{2}$ de validação de apenas 4,3 , enquanto a aderência da distribuição com valor default foi menos satisfatória porém também sem rejeição da hipótese nula ( $\chi^{2}$ de 5,7$)$. As médias das amostras obtidas em campo e simulada foram, respectivamente, iguais a 6,9 e 7,9 segundos, com um IC de 95\% para a diferença entre as médias igual a [-1,9 s; 0,0 s]. Como o zero se encontra no limite do intervalo, há indícios para se acreditar que as médias populacionais do tempo de espera simulado e observado são similares.



Figura 6: Validação - movimento conversão à direita

\section{COMPARAÇÃO COM CALIBRAÇÃO SIMULTÂNEA}

A fim de se comparar o método de calibração proposto com um dos métodos convencionais, realizou-se também a calibração do min_gap_time tendo como medida-alvo uma medida de desempenho do tráfego que se deseja estimar. No caso, como exemplo, a medida-alvo considerada foi o tamanho médio da fila, obtido a partir de observações a cada $15 \mathrm{~s}$.

Devido às diversas combinações capazes de gerar o mesmo tamanho médio de fila e à dificuldade (ou impossibilidade) de isolar o efeito da brecha crítica de cada movimento de conversão sobre essa medida-alvo, fixou-se o mesmo valor do parâmetro para ambos os movimentos e obteve-se um min_gap_time de 3,9 s para o mesmo cenário de calibração definido anteriormente, correspondendo a uma fila média igual à observada em campo, de 1,6 veículos. As distribuições dos tempos de espera obtidas em campo, no modelo calibrado pelo método proposto, no modelo calibrado pelo método convencional e na simulação com o parâmetro default para o movimento seguir em frente no cenário de validação são apresentadas na Figura 7, onde pode 
ser observada uma aderência um pouco menos satisfatória da distribuição referente à calibração do parâmetro pelo tamanho médio de fila, principalmente nas classes mais extremas, com $\chi^{2}$ de 9,3, enquanto o valor pelo método proposto foi de 7,9. A hipótese nula de aderência entre a distribuição default e a de campo foi rejeitada com um $\chi^{2}$ de 55,1.

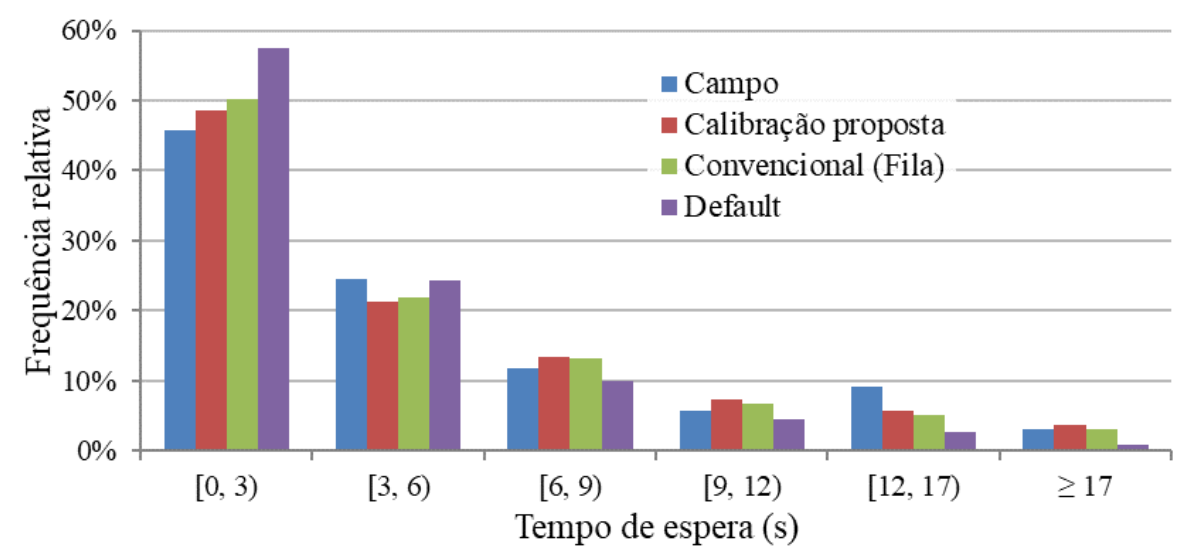

Figura 7: Comparação dos métodos de calibração - movimento seguir em frente

Como a medida-alvo proposta é impactada basicamente pelo processo de aceitação de brechas, nota-se que o método com base no tamanho médio de fila pode gerar resultados menos precisos devido a fatores externos como padrão de chegada dos veículos na via secundária, espaçamento entre eles e seus perfis de aceleração, correspondendo a uma modelagem menos representativa da realidade. Os resultados de ambos os métodos de calibração referentes à conversão à direita foram os mesmos, já que os valores ajustados para o parâmetro foram iguais. No caso de interseções onde a fila da aproximação secundária é maior e mais variável, há uma maior sensibilidade do min_gap_time sobre essa medida de desempenho, o que pode acarretar em diferenças maiores nos valores calibrados pelos diferentes métodos.

\section{CONCLUSÕES}

Embora a literatura apresente diversos trabalhos publicados sobre aceitação de brechas em interseções não semaforizadas, relativamente pouca atenção tem sido dada à modelagem por meio da microssimulação. Este trabalho propõe um método de calibração e de validação do modelo RP do VISSIM que tem como medida-alvo o tempo de espera do veículo que aguarda por uma brecha. Embora tenha se utilizado o VISSIM, o método proposto pode ser aplicado em outros simuladores cujos modelos de aceitação de brechas também são baseados em parâmetros de brecha crítica, como o TransModeler e o Aimsun.

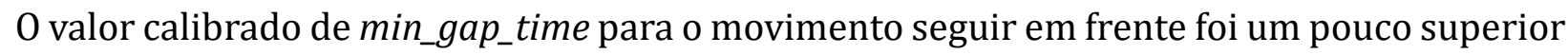
ao da conversão à direita, corroborando o conhecimento prévio. Ambos os valores foram superiores ao valor default do VISSIM, sugerindo que os motoristas do cruzamento em análise apresentaram perfil mais cauteloso quando comparado ao perfil padrão adotado no microssimulador.

Na etapa de validação, a hipótese de que os dados observados de tempo de espera se aderem bem ao modelo não pôde ser rejeitada. Além disso, as médias dos tempos de espera modelados para ambos os movimentos foram similares às observadas em campo, com uma leve superestimação para o caso do movimento de conversão à direita. Para a obtenção de um intervalo de 
confiança e uma segmentação do tempo de espera em classes mais estreitas e uma maior sensibilidade da estatística $\chi^{2}$, pode-se coletar mais observações em campo. Destaca-se também a importância de estabelecer um critério que combine ambas as medidas de ajuste adotadas $\left(\chi^{2}\right.$ e diferença entre as médias) a fim de tornar a calibração mais objetiva.

O comparativo realizado entre os métodos de calibração considerados indicou que o ajuste feito com base em medidas de desempenho do tráfego, como tamanho médio de fila, pode gerar resultados menos precisos, já que tais medidas dependem de outras variáveis como a demanda e o espaçamento entre os veículos. 0 problema pode ser agravado nos casos de filas mais extensas e variáveis e na calibração de mais de um parâmetro, pois pode resultar em combinações de valores inadequados dos parâmetros, mesmo que as diferenças entre os valores da medida de desempenho simulados e observados em campo sejam pequenas. Dessa forma, propõe-se a ca-

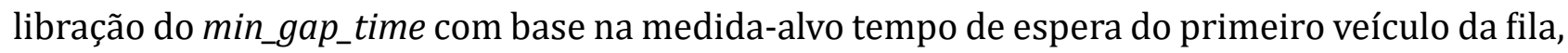
pois essa medida depende primordialmente do parâmetro.

Por se tratar de um método com base na distribuição de frequência de variáveis desagregadas, ele é relativamente exigente quanto ao esforço de coleta em campo. Por fim, sugere-se que em trabalhos futuros o método aqui proposto seja aplicado para outras interseções, possibilitando comparações entre os resultados para cruzamentos não semaforizados com diferentes características físicas e operacionais. A menos que as características da interseção em análise sejam semelhantes, os valores de min_gap_time aqui obtidos não devem ser utilizados, porém o método proposto pode ser aplicado, com possíveis adaptações.

\section{AGRADECIMENTOS}

Os autores agradecem ao CNPq o suporte financeiro por meio de bolsas e recursos de custeio.

\section{REFERÊNCIAS}

Brilon, W.; R. Koenig e R. J. Troutbeck (1999) Useful estimation procedures for critical gaps. Transportation Research Part A: Policy and Practice, v. 33, n. 3-4, p. 161-186. DOI:10.1016/S0965-8564(98)00048-2.

Depiante, V. S. e J. J. Galarraga (2012) Intervalos Criticos y Capacidad en Intersecciones no Semaforizadas de Tres Ramas. Congreso Panamericano de Ingeniería de Tránsito, Transporte y Logística, PANAM, Santiago, Chile.

Elefteriadou, L. (2014) An Introduction to Traffic Flow Theory. Ed. Springer, New York. DOI:10.1007/978-1-4614-8435-6.

Garber, N. J. e L. A. Hoel (2015) Traffic and Highway Engineering. 5. Ed. CL Engineering.

Jie, L.; H. V. Zuylen; Y. Chen; F. Viti e I. Wilmink (2013) Calibration of a Microscopic Simulation Model for Emission Calculation. Transportation Research Part C: Emerging Technologies, v. 31, n. 0, p. 172-184. DOI:10.1016/j.trc.2012.04.008.

Kyte, M.; M. Dixon e P. M. Basavaraju (2003) Why Field Measurements Differ from Model Estimates. Transportation Research Record: Journal of the Transportation Research Board, v. 1852, p. 32-39. DOI:10.3141/1852-05.

Lacerda, V. M. e M. M. Castro-Neto (2014) Considerações sobre a Calibração do Modelo de Car-following do VISSIM para Vias Arteriais Urbanas. Anais do XXVIII Congresso de Pesquisa e Ensino em Transportes, ANPET, Curitiba.

Lacerda, V. M. (2016) Estimação da Velocidade Média em Vias Urbanas com Uso do Microssimulador VISSIM. Dissertação (Mestrado em Engenharia de Transportes). Programa de Pós-Graduação em Engenharia de Transportes, Universidade Federal do Ceará, Fortaleza, CE.

Lidbe, A. D.; A. M. Hainen e S. L. Jones Jr. (2016) Comparative Study of Simulated Annealing and Genetic Algorithm for Calibration of Microsimulation Model. In: Transportation Research Board 95th Annual Meeting, Washington, USA.

Liu, P.; X. Q. H. Yu; W. Wang e B. Cao (2012) Development of a VISSIM Simulation Model for U-Turns at Unsignalized Intersections. Journal of Transportation Engineering, v. 138, n.11, p. 1333-1339. DOI:10.1061/(ASCE)TE.1943-5436.0000438.

Martin-Gasulla, M.; A. García e C. Llorca (2017) Headway Acceptance Decisions on Single-Lane Roundabouts in Spain. Critical and Follow Headway. In: Transportation Research Board 96 th Annual Meeting, Washington, USA.

Mohan, M. e S. Chandra (2016) Influence of Major Stream Composition on Critical Gap at Two-Way Stop-Controlled Intersections. In: Transportation Research Board 95th Annual Meeting, Washington, USA.

PTV (2016) VISSIM 9.0. Manual do Usuário. Plannung Transport Verkehr AG, Karlsruhe, Germany.

Raff, M. S. (1950) A Volume Warrant for Urban Stop Signs. Eno Foundation for Highway Traffic Control, Saugatuck, Connecticut.

Tarko, A. e Z. Tian (2003) Example Analysis and Handling of Uncertainty in the Highway Capacity Manual with Consideration of Traffic Diversion. Transportation Research Record: Journal of the Transportation Research Board, v. 1852, p. 40-46. DOI:10.3141/1852-06. 
TRB (2010) Highway Capacity Manual. Transportation Research Board, v. 3, Washington.

Viti, F.; B. Wolput; C. M. J. Tampère e P. Vandervelden (2013) Dynamic Modeling of VISSIM's Critical Gap Parameter at Unsignalized Intersections. Transportation Research Record: Journal of the Transportation Research Board, v. 2395, p. 12-20. DOI:10.3141/2395-02. 